\title{
Small-Scale Hydrodynamics of Feeding Appendages of Marine Animals
}

\author{
M.A.R. Koehl \\ Dept. of integratize Biology $\bullet$ University of California $\bullet$ Berkeley, California 94720 USA
}

M any animals in the ocean use appendages bearing arrays of hairs to capture molecules from the surrounding fluid (e.g. feathery gills take up oxygen; olfatory antennae capture odorants), to capture food particles (e.g. hairy suspension-feeding appendages catch single-celled algae), or to move the fluid around them (e.g. setulose appendages are used to swim or create ventilatory currents). Since hairy little appendages serve such important biological functions in animals from so many phyla, we have been trying to elucidate the basic rules governing how they all work.

\section{Hydrodynamics of "Hairy Little Legs"}

The performance of all the functions mentioned above (e.g. capturing molecules or particles; moving water) depends on how the arrays of hairs interact with the water around them (e.g. Koehl, 1981; 1995; Childress, et al., 1987). Therefore, the first step in analyzing how hairy appendages work is to figure out how fluid moves around and through them. The Reynolds number (Re) of a structure moving through a fluid represents the relative importance of inertial to viscous forces determining how the fluid moves; $R e=\rho L U / \mu$, where $L$ is a linear dimension of the structure,

... the first step in analyzing how hairy appendages work is to figure out how fluid moves around and through them.
Cheer and Koehl, 1987; 1988; Loudon, et al., 1994; Koehl, 1995). In this Re range viscosity is very important in determining flow patterns (although we cannot ignore the effects of inertia at the upper end of this Re range, Cheer and Koehl, 1987; 1988; Koehl, 1992; 1995). Since humans operate at high Re (approximately $10^{\circ}$ when swimming), we cannot trust our intuitions when considering the viscous flow around arrays of little hairs.

In order to understand how arrays of hairs capture molecules or particles, or push fluids around, the first thing that we need to figure out is whether fluid flows through the gaps between the hairs in an array, or flows around the sides of the array rather than through it. We have defined the "leakiness" of an array of hairs as the proportion of the water encountering the gap between adjacent hairs that actually flows through the gap (Cheer and Koehl, 1987). The leakiness of a hair-bearing structure determines whether or not the structure can function as a filter, and it also affects the flux of molecules to hair surfaces and the ability of the appendage to generate thrust or lift (e.g. Koehl, 1995; 1996a). Since there is diversity in the the size, structure, and behavior of hair-bearing appendages, another important piece of the puzzle that we have to address
$\mathrm{U}$ is fluid velocity relative to it, and $\rho$ and $\mu$ are the density and viscosity (resistance to being sheared) of the fluid (e.g. Vogel, 1994). At high Re (e.g. large, rapidlymoving structures), inertial forces predominate and flow is messy and turbulent, whereas at low $\operatorname{Re}$ (e.g. small, slowly-moving structures), viscosity damps out disturbances in the fluid and flow is smooth and orderly. When fluid flows past a solid surface, the fluid in contact with the surface does not slip relative to the surface and a velocity gradient develops between the surface and the freestream flow. At low Re, this layer of sheared fluid between the surface of a moving structure and the still surrounding fluid is thick relative to the dimensions of the structure (e.g. Koehl, 1981; 1995). If we calculate the Re at which the hairs on the types of appendages listed above operate (using hair diameter for $\mathrm{L}$ ), we find that they range between $10^{-5}$ and 10 (Rubenstein \& Koehl, 1977; Koehl and Strickler, 1981; is how the morphology and motion of a hairy leg affect its leakiness (Koehl, 1983; 1995; 1996b; Loudon, et al., 1994).

A general model of flow between neighboring hairs permits us to examine how the size, spacing, and speed of an array of hairs affect its leakiness (Cheer and Koehl, $1987 ; 1988)$. At small hair sizes $\left(\operatorname{Re}=10^{-5}\right.$ to $\left.10^{-3}\right)$, arrays of hairs have very low leakiness (i.e. only a small proportion of the water or air encountered actually goes through the gaps between hairs, while most flows around the array) and function like non-porous paddles. In contrast, from $\operatorname{Re}$ of $10^{-2}$ to 1 , a transition in leakiness occurs: a structure that functioned like a paddle at low speed and small size becomes a leaky sieve at faster speed or larger size. We can apply these general principles to study how "hairy little legs" (such as the feeding appendages of copepods) work, and how their structure and behavior affect their performance. 


\section{Copepod Feeding Appendages}

Calanoid copepods are abundant planktonic crustaceans that play a critical link in many marine food webs between single-celled algae and higher trophic levels such as fish. Since copepod feeding is so ecologically important, many studies measuring copepod feeding rates and selectivity have been conducted (reviewed in Koehl, 1984). To complement these studies, we have been working to figure out the physical mechanisms copepods use to catch particles like single-celled algae.

High-speed microcinematography of seawater labeled with dye and released from miciopipettes near the animals while they were catching food particles revealed the appendage and water motions involved in copepod feeding (Koehl and Strickler, 1981; Koehl and Paffenhöfer, unpubl. data). The last stage in particle capture is performed by a pair of setulose appendages, the second maxillae (M2's; Fig. 1), that fling apart from each other and then squeeze back together again. Some species perform this capture motion with their setae (hairs) operating at Re of order 1, whereas others do it at hair Re as low as $10^{-2}$ (Koehl, 1981; Koehl and Strickler, 1981; Koehl, 1992; 1995). Remember, this represents the critical Re range in which the transition occurs between non-leaky paddle-like behavior and leaky sieve-like function.

Analysis of the motions of M2's, particles, and dye in the movies of feeding copepods revealed that species such as Centropages velificatus that have coarsely-meshed M2's (Fig. 1) whose setae (hairs) operate at $\operatorname{Re}=1$, have leaky M2's and filter their food from the water during
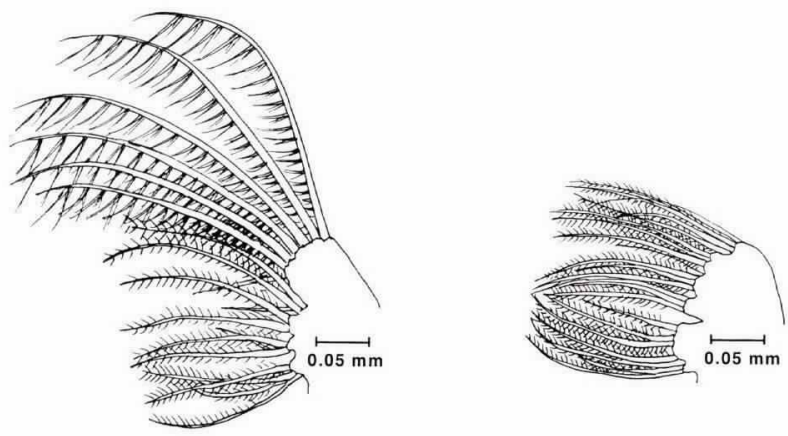

\section{Centropages velificatus}

\section{Temora stylifera}

Figure 1. Diagrams of second maxillae from the calanoid copepods Centropages velificatus (whose setae operate at Reynolds numbers of order 1) and of Temora stylifera (whose setae operate at Reynolds numbers of order $\left.10^{-2}\right)$.

the squeeze (Koehl, 1995). In contrast, other species such as Temora stylifera that have finely-meshed (Fig. 1), slowly-moving $M 2$ 's whose setae operate at $\mathrm{Re}=$ $10^{-2}$, have paddle-like $\mathrm{M} 2^{\prime}$ s that capture food by drawing a parcel of water containing an alga towards the mouth during the fling (Koehl 1981; Koehl and Strickler, 1981; Koehl, 1995). Thus, even though their M2 feeding motions look qualitatively similar, the physical mechanisms by which these two copepods capture food are different because they operate at Re above and below the transition from paddle to sieve. These copepod M2's provide examples of hairy appendages that look similar to each other and that move qualitatively in the same way, but that capture algal cells by different mechanisms during that motion simply because they operate at Reynolds

We have been using physical models of copepod M2's to tease out whether leakiness is affected by the coarseness of the mesh of hairs on the M2's as well as by their speed. Like mathematical models, physical models permit us to vary only one parameter at a time to quantify its effects while holding all the other vari-

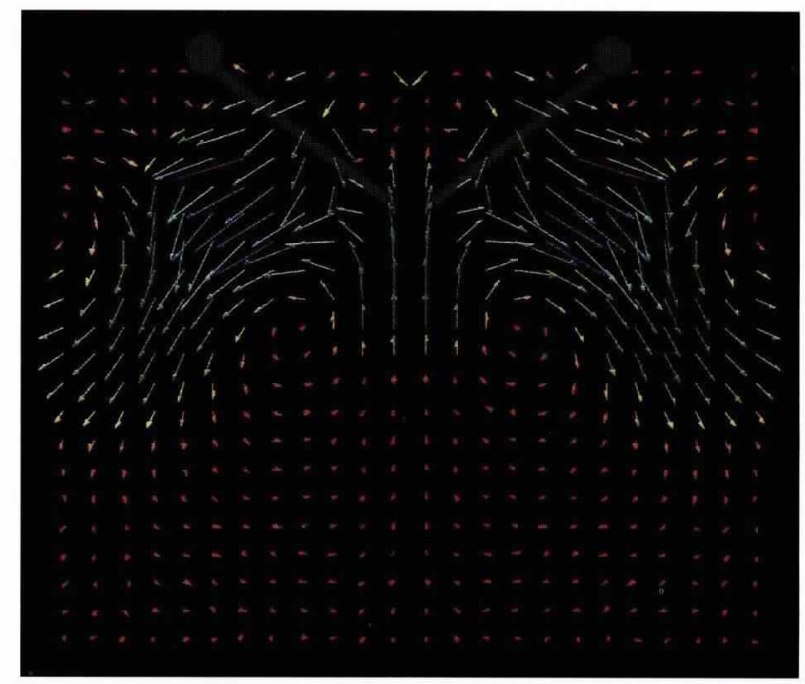

Figure 2. Diagram of the net displacement of water produced by the "flingand-squeeze" motion of a pair of food-particle-capturing appendages (second maxillae, M2's) of the calanoid copepod Centropages velificatus (arrows indicate water direction and their color represents distance moved: blue $460 \mu \mathrm{m}$, green $\sim 230 \mu \mathrm{m}$, red $<1 \mathrm{~mm})$. The gray bars indicate the positions of the M2's (390um long) after the fling-and-squeeze is completed, and the gray circles represent the hinges between the M2's and the body surface of the animal. We are looking down on the anterior end of a copepod that is vertical in the water; the body of the animal is at the top of the picture, the M2's are on the ventral surface, and the mouth is midway between the M2's. During the fling, the M2's rotate away from each other, and during the squeeze, they rotate back towards each other. Some copepods operate their M2's so slowly that little water flows through the array of setae (hairs) on an $\mathrm{M} 2$, which therefore functions like a paddle moving water containing food particles towards the mouth. In contrast, C. velificatus moves its M2's more rapidly and water flows through rather than around the array of hairs on each M2. These leaky M2's can filter particles from the water moving through them, whereas paddle-like M2's cannot. As this diagram indicates, water is drawn towards the mouth and is passed laterally through the M2's when a C. velifacatus does a fling-and-squeeze. This flow visualization was made using a dynamically-scaled physical model of a pair of $C$. velificatus M2's attached to a body surface, and this image was produced by T. Cooper using the particle image velocimetry program described by Cowan and Monismith (1997). 
ables constant (Koehl, 1992; 1995); such manipulations are not possible in comparative studies with real animals. If a physical model and the propotype M2's are geometrically similar and operate at the same Re, then the ratios of the velocities and the forces at comparable positions in the flow field around the model are the same as those around the M2 (Vogel, 1994). Therefore, we can slowly flap large models of copepod M2's in high viscosity mineral oil at the Re used by the copepods, and we can also change the speeds of the models to make them operate at different Re used by other

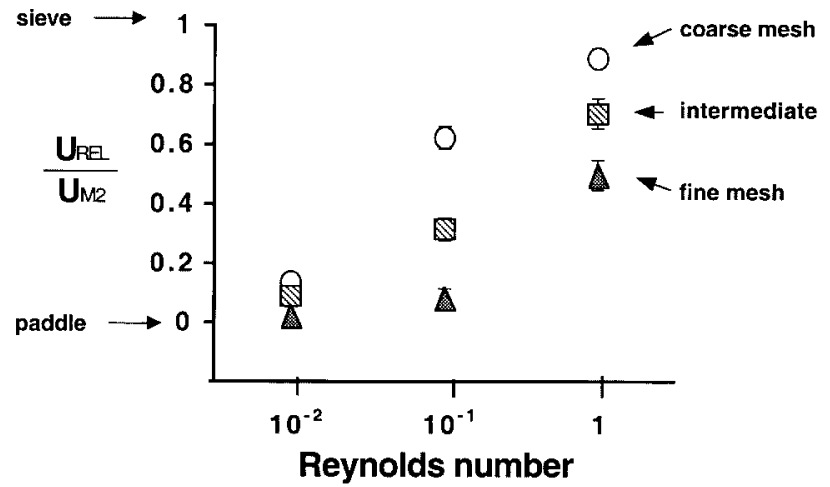

Figure 3. Flow through the middle of the array of hairs on models of the second maxillae of calanoid copepods: Centropages velificatus (coarse mesh of hairs on the second maxillae; indicated by circles), Eucalanus pileatus (intermediate mesh; shown by squares), and Temora stylifera (fine mesh; indicated by triangles). $U_{R E L}$ is the velocity of fluid between the hairs relative to the hairs, and $U_{m 2}$ is the speed of the second maxilla; the ratio $U_{R E L} / U_{M 2}$ is an indication of leakiness (values near one are leaky and sievelike, while values near zero are un-leaky and paddle-like). Even though $C$. velificatus operate their second maxillae at seta Reynolds numbers of order 1 , while the other two species operate at Re of order $10^{-2}$, we could run the models at any Re we chose. These experiments showed that at a Reynolds number of $10^{-2}$, the coarseness of the mesh of hairs on a second maxilla makes no difference to its leakiness, whereas at Reynolds numbers of $10^{-1}$ and 1, coarsely-meshed appendages are leakier than finely-meshed ones (error bars indicate one standard deviation, $n=3$ to 12).

species. By videotaping the paths of neutrally-buoyant marker particles in the fluid, we have measured the flow produced during the fling and squeeze (Fig. 2) and we have used these data to calculate the M2 leakiness. From such experiments we learned that the coarser the mesh, the leakier the $M 2^{\prime} s$ at $\operatorname{Re} \geq 10^{-1}$, but that at $\operatorname{Re}=$ $10^{-2}$, mesh coarseness has no effect on leakiness (Fig. 3). Although this result-that the morphology of an appendage only affects its performance at some Re, but not at others-seems non-intuitive, it was predicted from the basic physics of how fluids flow around cylinders (Cheer and Koehl, 1987; 1988; Koehl, 1992; 1995). We are now applying the same principles to investigate molecule capture by "hairy little noses" (e.g. we are studying the hydrodynamic design of olfactory antennules of a variety of crustaceans) (Koehl, 1996b).

\section{REFERENCES}

Cheer, A. Y. L. and M. A. R. Koehl, 1987: Paddles and rakes: Fluid flow through bristle appendages of small organisms. J. Theor. Biol., 129, 17-39.

Cheer, A. Y. L. and M. A. R. Koehl, 1988: Fluid flow through filtering appendages of insects. I.M.A. J. Math. Appl. Med. Biol., 4, 185-199.

Childress, S., M. A. R. Koehl, and M. Miksis, 1987: Scanning currents in Stokes flow and the efficient feeding of small organisms. J. Fluid Mech., 177, 407436.

Cowen, E.A., and S.G. Monismith, 1997: A hybrid digital particle tracking velocimetry technique. Experiments in Fluids, 22, 199-211.

Koehl, M. A. R., 1981: Feeding at low Reynolds number by copepods. Lectures in Mathematics in the Life Sciences, 14, 89-117.

Koehl, M. A. R., 1983: The morphology and performance of suspension-feeding appendages. J. Theor. Biol., 105, 1-11.

Koehl, M. A. R., 1984: Mechanisms of particle capture by copepods at low Reynolds number: Possible modes of selective feeding. In: Trophic Interactions Within Aquatic Ecosystems. D. L. Meyers and J. R. Strickler, eds., Westview Press, 135-160.

Koehl, M. A. R., 1992: Hairy little legs: Feeding, smelling, and swimming at low Reynolds number. Fluid Dynamics in Biology. Contemporary Mathematics, 141, 33-64.

Koehl, M. A. R., 1995: Fluid flow through hair-bearing appendages: Feeding, smelling, and swimming at low and intermediate Reynolds number. Soc. Exp. Biol. Symp., 49, 157-182.

Koehl, M. A. R., 1996a: When does morphology matter? Ann. Rev. Ecol. Syst., 27, 501-542.

Koehl, M. A. R., 1996b: Small-Scale fluid dynamics of olfactory antennae. Mar. Fresh. Behav. Physiol., 27, 127-141.

Koehl, M. A. R. and J. R. Strickler, 1981: Copepod feeding currents: Food capture at low Reynolds number. Limnol. Oceanogr., 26, 1061-1073.

Loudon, C., B. A. Best, and M. A. R. Koehl, 1994: When does motion relative to neighboring surfaces alter the flow through an array of hairs? J. Exp. Biol., 193, 233254.

Rubenstein, D. I. and M. A. R. Koehl, 1977: The mechanisms of filter feeding: Some theoretical considerations. Amer. Natur., 111, 981-994.

Vogel, S., 1994: Life in Moving Fluids: The Physical Biology of Flow, $2^{\text {wid }}$ Edition. Princeton University Press, Princeton. 